i-DUST 2010, $02003(2011)$

DOI: 10.1051 idust/201102003

(C) Owned by the authors, published by EDP Sciences, 2011

\title{
Characterisation of magnetic field fluctuations at different locations within the Laboratoire Souterrain à Bas Bruit using a new SQUID magnetometer prototype
}

\author{
V. Andrieux ${ }^{1}$, M. Auguste ${ }^{2}$, D. Boyer ${ }^{2}$, A. Cavaillou ${ }^{2}$, C. Clarke ${ }^{1}$, P. Febvre $^{3}$, \\ S. Gaffet ${ }^{2}$, S. Henry ${ }^{1}$, H. Kraus ${ }^{1}$, A. Lynch ${ }^{1}$, V. Mikhailik ${ }^{1}$, M. McCann ${ }^{1}$, \\ E. Pozzo di Borgo ${ }^{2}$, C. Sudre ${ }^{2}$, and G. Waysand ${ }^{2}$ \\ ${ }^{1}$ University of Oxford, Denys Wilkinson Building, Keble Road, Oxford, OX1 3RH, UK \\ ${ }^{2}$ Laboratoire Souterrain à Bas Bruit, UNS/CNRS/OCA, 84400 Rustrel, France \\ ${ }^{3}$ IMEP-LAHC, CNRS UMR5130, Université de Savoie, 73376 Le Bourget du Lac Cedex, \\ France
}

\begin{abstract}
We have carried out a series of magnetic field measurements using a portable three-axis SQUID magnetometer at the Laboratoire Souterrain à Bas Bruit (LSBB), Rustrel, France. The magnetometer was originally developed as part of the cryoEDM neutron electric dipole moment experiment [1], where we need to monitor drifts in the magnetic field at a level of $\sim 0.1 \mathrm{pT}$. The cryoEDM SQUID system is a 12-channel magnetometer designed to operate in a large cryostat with extensive magnetic shielding [2]. We have tested smaller prototype systems during a series of trips to LSBB [3], primarily to test the SQUIDs, and control and DAQ electronics in a low noise environment. However this investigation also provided an opportunity to characterise the magnetic environment at different locations within the LSBB complex. We monitored the magnetic field at various positions inside the underground laboratory, including the Capsule, the Galerie Anti-Souffle (GAS) and the Galerie Gaz-Brûlés (GGB). We recorded several hours of data at each location to compare with that recorded at the same time by the LSBB [SQUID] ${ }^{2}$ system permanently installed in the Capsule, and from this we have characterised the relative amplitudes of magnetic field fluctuations in the different locations. SQUID resets are corrected using software, but as this process is not perfect an accurate comparison can only be done during stable periods. Software development and data analysis are still in progress. Preliminary analysis suggests the magnetic field measured in the Capsule is approximately $75 \%$ that measured in the GAS and GGB.
\end{abstract}

\section{INTRODUCTION}

The Oxford cryoEDM group have designed and constructed a 12-channel SQUID magnetometer as part of the cryoEDM neutron electric dipole moment experiment [1]. The low noise environment at LSBB is the ideal place to test such a system. In 2006 we carried out a series of measurements at LSBB to characterise our system and develop the software to correct for effects such as SQUID resets and crosstalk [2]. Since then we have further improved our system by upgrading the DAQ system to a new continuous digitisation system.

In 2009 we returned to LSBB and took further measurements using a 3-axis magnetometer. This was used to monitor the magnetic field at different locations in the underground complex (shown in Figure 1) with the aim of characterising the noise and magnetic shielding factors of the laboratory, by comparing the signal measured by our system with that recorded by the $[\mathrm{SQUID}]^{2}$ system permanently installed in the LSBB Capsule [4].

This is an Open Access article distributed under the terms of the Creative Commons Attribution-Noncommercial License 3.0, which permits unrestricted use, distribution, and reproduction in any noncommercial medium, provided the original work is properly cited. 


\section{i-DUST 2010}

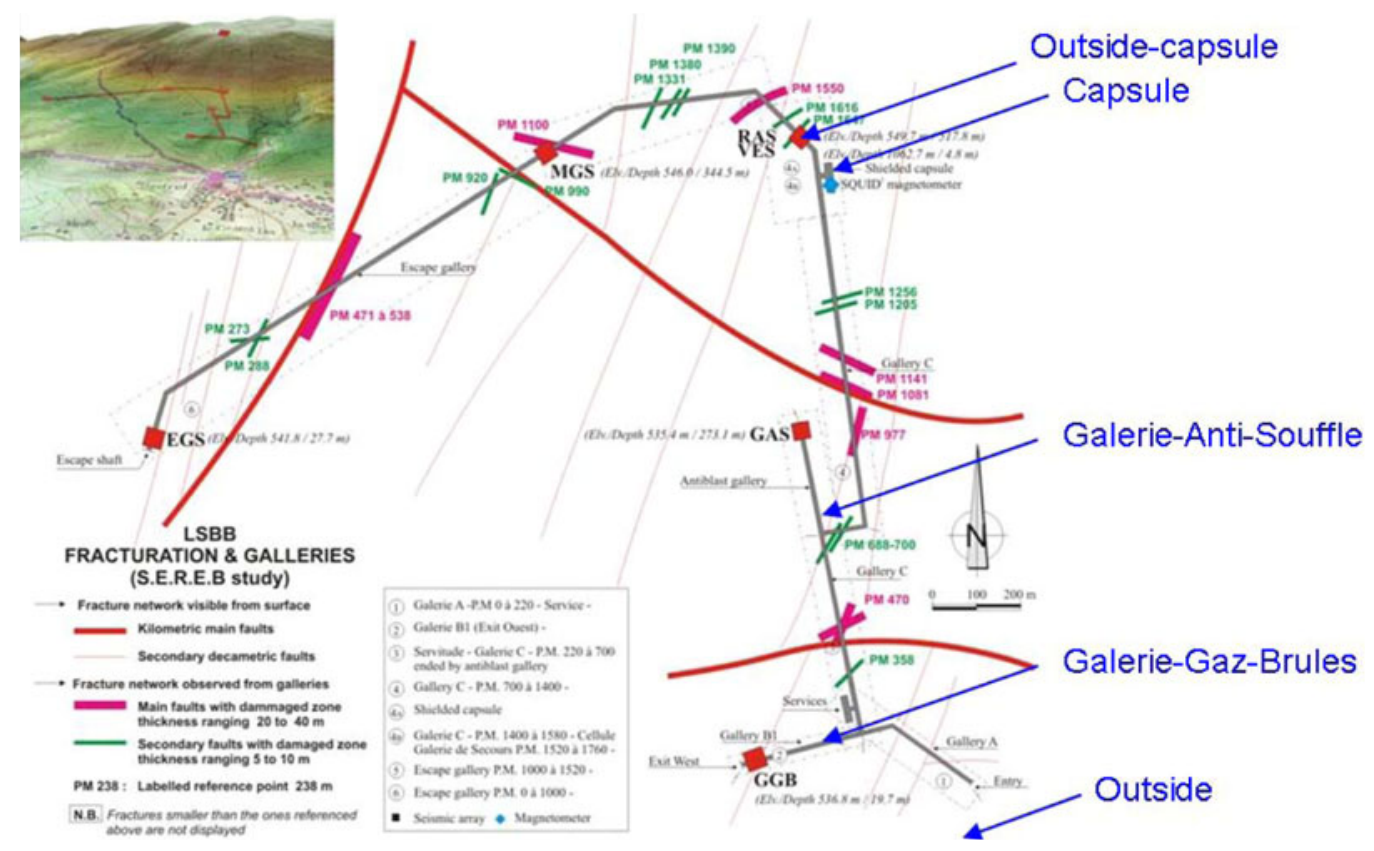

Figure 1. The locations of magnetometry measurements using the 3-axis setup, superimposed on a map of the LSBB seismometers [3].

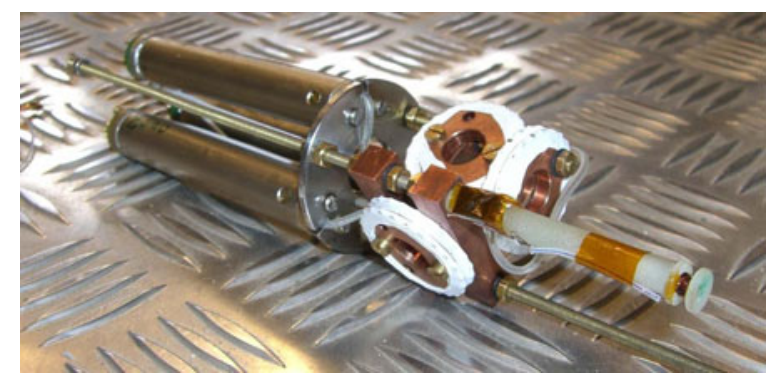

Figure 2. The 3-axis SQUID magnetometry system used for these measurements.

The 3-axis setup consists of three orthogonal pick-up loops made from niobium wire connected to SQUIDs. The three channels are here referred to as Z, NS and EW, as orientated to measure the vertical, North-South, and East-West components of the magnetic field. This is shown in Figure 2. A calibration coil consisting of 200-300 turns of copper wire on a 1mm former was mounted about 60mm away from the pick-up loops. The setup is mounted on a dipstick and lowered into a helium dewar.

The SQUIDs were controlled using a STAR-Cryoelectronics PCI1800 unit with PFL800 preamplifier. The output of the SQUID system was recorded using a continuous digitisation system on a card in the PCI bus of the data acquisition PC. This was sampled at $1 \mathrm{kHz}$. The continuous digitisation is an improvement on the system used for measurements in 2006. By eliminating dead time, we aim to use this to do a complete correction of SQUID resets. The new system also avoids the potentially noisy Camac crate used for previous measurements.

Where measurements were taken in the Capsule, the PFL800 and PCI1800 units were installed close to the dewar inside the Capsule. DC power $( \pm 12 \mathrm{~V})$ was supplied by a $10 \mathrm{~m}$ screened power lead leading 


\section{Andrieux et al.: Characterisation of magnetic field fluctuations at different locations}

to power supply just outside the Capsule. The signals were taken to the data acquisition card in the PC (also outside the Capsule) using screened cables. The SQUID control signals were delivered by optical fibre.

This field trip provided the first opportunity to test the new system, with continuous digitisation, in a low noise environment. This showed it worked well, and provided the data needed to develop software to correct SQUID resets. This is described in the following section.

\section{DATA CORRECTIONS}

Before the recorded data could be used to inspect the magnetic field, it is first necessary to correct the effect of SQUID resets. These occur whenever the output voltage came close to the maximum or minimum of its range, the control electronics would then 'reset' the system by temporarily opening the feedback loop so the output jumps an integer number of flux quanta. These resets can be corrected by identifying when they occur and offsetting the baseline accordingly. As resets occurred frequently (typically 3 to 15 per hour depending on the channel and the time), without this correction the maximum length of continuous data available would be too short for a useful analysis.

The data is divided up into events of $512 \mathrm{~ms}$ length. Converting the raw data files, containing the SQUID output voltage, to a file giving the average magnetic field in $\mathrm{nT}$, averaged over each event, was done in a series of steps:

- Calculate the average voltage over the 512 data points in an event.

- Calculate the number of net resets in an event. A reset is identified by a jump of more than a given magnitude, between a number of data points. One positive (from $\sim-10 \mathrm{~V}$ to $\sim 0 \mathrm{~V}$ ), or two positive and one negative reset would both give a Net Resets of +1 .

- Calculate the absolute baseline [V] for each event, by adding or subtracting the reset magnitude to the average value for each event depending on the value of net resets.

- Calculate the absolute baseline ${ }^{1}[n T]$ by multiplying the absolute baseline [V] by the calibration factor.

The magnitude of SQUID resets was calculated by fitting a straight line to the SQUID output over a sample period before and after the reset, avoiding the overshoot and exponential decay immediately following the reset. The reset magnitude is then the vertical difference between the lines. This is illustrated in Figure 3, the values calculated from a selected number of resets are 7.558 V (Z), 5.556 (NS). These correspond to jumps of $3 \Phi_{0}$ and $2 \Phi_{0}$ (note the voltage corresponding to $1 \Phi_{0}$ is different for each channel, depending on the SQUID, and the inductance of the pick-up loop). The EW SQUID resets by $-5.479\left(2 \Phi_{0}\right)$ when the output approached $+10 \mathrm{~V}$ and $+8.272\left(3 \Phi_{0}\right)$ when it approaches $-10 \mathrm{~V}^{2}$

The calibration factor [in $\mathrm{V} / \mathrm{nT}$ ] is determined from the signal applied to a calibration coil consisting of 200-300 turns wound on a $1 \mathrm{~mm}$ former. This is modelled as a magnetic dipole, but as the loops are off axis, and orientated in different planes, the magnetic field must be calculated numerically.

Note for the 3-axis data, no correction for the crosstalk between loops was necessary as the crosstalk between orthogonal loops is negligible.

This reset correction procedure is not necessary for the $[\text { SQUID }]^{2}$ data as this data is recorded in a different way. The SQUIDs are operated in a lower range so the magnitude of one flux quantum is much less. The signals are digitised with a high resolution (26-bit) ADC so the full range of the magnetometer is within the $\pm 10 \mathrm{~V}$ range of the data acquisition. Therefore there are no resets as seen in the Oxford data, however rapid changes in the field may cause flux jumps.

\footnotetext{
${ }^{1}$ Note this is not the absolute value of the magnetic field (which one cannot measure with this sort of SQUID system), but a parameter with a constant offset for the entire dataset.

${ }^{2}$ This was true for all resets recorded with this SQUID.
} 
Graph

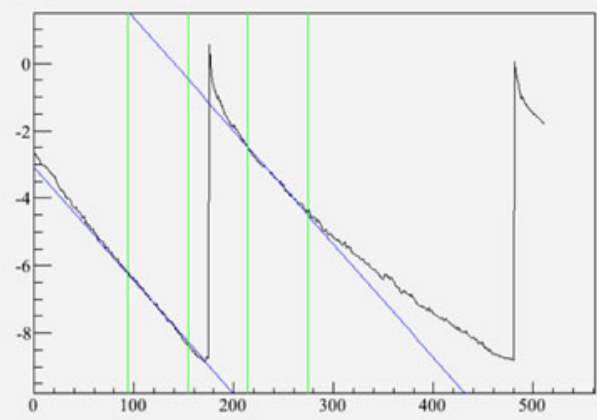

h1_1

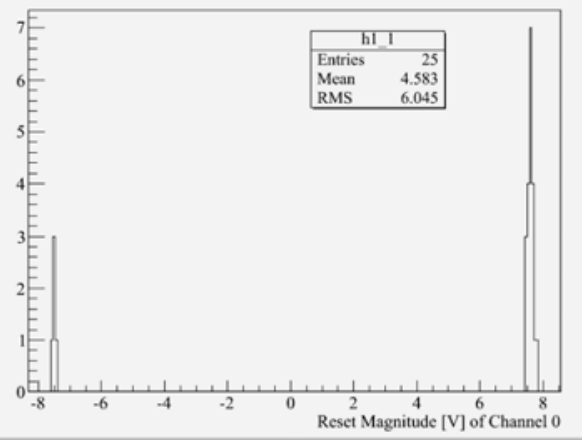

Figure 3. The magnitude of a reset is calculated by fitting a straight line to the SQUID data before and after the reset. The histogram shows the magnitude of resets for a selected sample for channel Z.
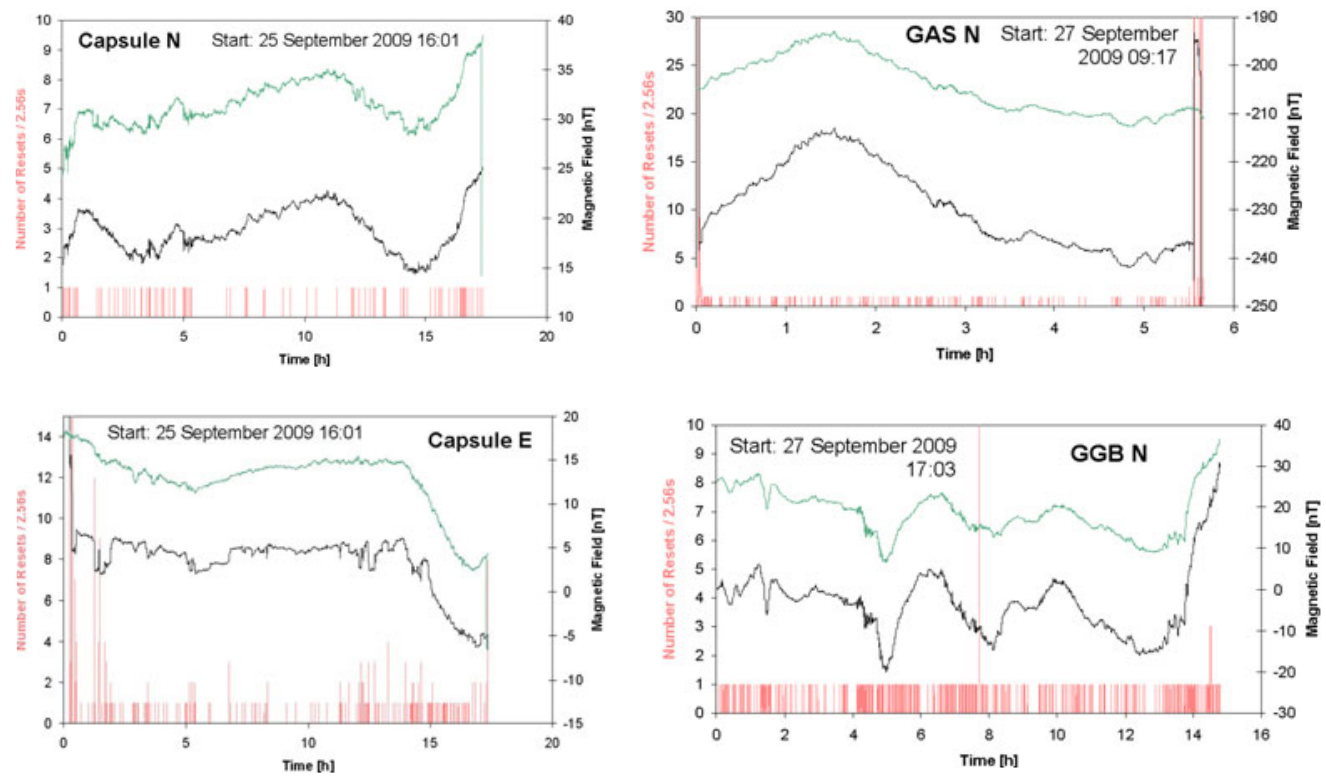

Figure 4. Magnetic field measurements taken by the Oxford 3-axis setup (lower black line) in the Capsule (NS and $\mathrm{EW}$ ) and Galerie Gaz Brules and Galerie Anti-Souffle (N), compared to the signal recorded in the Capsule by the [SQUID] ${ }^{2}$ system (upper green lines). The lines have been offset for clarity as the absolute value is arbitrary. Also shown in red is the number of SQUID-resets recorded by the Oxford system (in $2.56 \mathrm{~s}$ bins), which gives an impression of the stability of the system.

\section{DATA ANALYSIS}

\subsection{Magnetometry data and comparison with [SQUID]2 data}

Figure 4 shows the resulting magnetic field signal measured with the [SQUID] ${ }^{2}$ system, and the Oxford 3 -axis setup after the corrections described in section 2 for a selection of data sets. Clearly both systems are measuring the external field, although this is disrupted by flux jumps and resets which are not perfectly corrected. It is interesting to note that the EW signal measured by the [SQUID] ${ }^{2}$ system is smooth, whereas the Oxford signal has many abrupt jumps due to resets or flux jumps; however the 


\section{Andrieux et al.: Characterisation of magnetic field fluctuations at different locations}

situation for the $\mathrm{Z}$ signal is reversed - the Oxford signal is smooth but the [SQUID] ${ }^{2}$ system shows abrupt jumps. This suggests that the rapidly changing signals are not a feature of the external field.

We took measurements in 5 different locations. The measurements recorded outside were very noisy with many flux jumps. This was also the case in the outside-capsule location due to the presence of nearby power lines. The other locations: the capsule, galerie anti-souffle, and galerie gaz-brules were very quiet environments limited only by geomagnetic fluctuations.

A key aim of this analysis is to determine the magnitude of the attenuation of the DC magnetic field at different locations at LSBB (in effect the 'shielding factor' of the laboratory). In principle this can be achieved by comparing the magnitude of the field fluctuations recorded by the [SQUID] ${ }^{2}$ system in the Capsule, with those measured by the Oxford system in a remote location. As the dominant magnetic fluctuations are produced by currents in the ionosphere $(\sim 300 \mathrm{~km}$ away), it is reasonable to assume the signal measured in the Capsule will be the same as that measured in other locations $(\sim 1 \mathrm{~km}$ away), but attenuated by the extra rock and metal surrounding it.

Unfortunately this task is not as straightforward as originally thought, as the many SQUID resets and flux jumps in both data sets mean we cannot simply rescale one record to fit another.

We plan to develop software to identify flux jumps, and thus identify the stable regions where the signals can be compared. As a very preliminary result, we present in Table 2 the results derived by selecting regions which gave good fit.

We define the attenuation factors as the ratio of the magnitude of the magnetic field fluctuations measured in the Capsule $\left\langle B_{C A P}\right\rangle$, to that measured in the Galerie Anti-Souffle $\left\langle B_{\mathrm{GAS}}\right\rangle$, and the Galerie Gaz-Brules $\left\langle B_{G G B}\right\rangle$.

$$
A_{G A S}=\frac{\left\langle B_{C A P}\right\rangle}{\left\langle B_{G A S}\right\rangle} \quad A_{G G B}=\frac{\left\langle B_{C A P}\right\rangle}{\left\langle B_{G G B}\right\rangle} .
$$

Note that these factors are defined for the quasi-DC field, the magnitude of fluctuations seen over several hours. This does not represent the ratio of the absolute magnetic field (which one cannot measure using SQUIDs). We expect the attenuation to increase with frequency but we assume it is frequency independent for this analysis.

To determine these ratios we compare the magnetic fluctuations recorded in the two locations over the same period of at least a few hours. This obviously necessitates two magnetometers. However we cannot simply compare the signals from the two magnetometers as the dominant difference is due to the imperfect calibration in one or both systems (we believe the largest error is in the Oxford system). ${ }^{3}$ In addition as SQUIDs only measure relative changes in the magnetic flux, there is an arbitrary offset on each dataset.

We determine the attenuation by taking the ratio of the signal measured by the Oxford system in the Capsule, to that measured by the [SQUID] ${ }^{2}$ system in the Capsule; and dividing this by the ratio of the signal measured on another day by the Oxford system in the remote location, to that measured on that day by the $[\mathrm{SQUID}]^{2}$ system in the Capsule:

$$
A_{G A S}=\frac{\left\langle B_{C A P}\right\rangle}{\left\langle B_{G A S}\right\rangle}=\frac{\left\langle B_{C A P}^{\text {oxday1 }}\right\rangle}{\left\langle B_{C A P}^{[\mathrm{SQ}] \text { day } 1}\right\rangle} \frac{\left\langle B_{C A P}^{[\mathrm{SQ}] \text { day } 2}\right\rangle}{\left\langle B_{G A S}^{\text {oxday2 }}\right\rangle} .
$$

For the Capsule measurement, the two systems are separated only by $\sim 1 \mathrm{~m}$ so we assume they measure the same field. The ratios are determined by a process which subtracts the offset and averages over the entire dataset; this is done as follows.

${ }^{3}$ The magnetic field can be modified by the superconducting films and coils on the SQUID chips, due to the Meissner-Ochsenfeld effect. 
Table 1. Preliminary values for $\alpha$-the relative amplitude of the Oxford signal compared to the [SQUID] $]^{2}$ signal (see text) for selected short periods for the three magnetic field component. The deviation from $\alpha=1$ for the capsule data represents the difference in the calibration factors for the two systems. The values for the other locations are determined by this, as well as the additional attenuation of the field in the capsule.

\begin{tabular}{|l|ccc|}
\hline \multirow{2}{*}{ Location } & \multicolumn{3}{|c|}{$\alpha$} \\
\cline { 2 - 4 } & $\mathrm{Z}$ & $\mathrm{NS}$ & $\mathrm{EW}$ \\
\hline Capsule & 0.769 & 0.811 & 1.308 \\
\hline Galerie Anti-Souffle & 0.563 & 0.671 & 0.881 \\
\hline Galerie Gaz-Brules & 0.560 & 0.611 & 0.985 \\
\hline
\end{tabular}

Table 2. Preliminary results for the attenuation factor A (given as percentages) - the magnitude of the magnetic field fluctuations measured in the Capsule compared to that measured in the Galerie Anti-Souffle, and the Galerie Gaz-Brules. If these values are accurate it suggests the additional rock and steel surrounding the capsule attenuates the field to about $75 \%$ of the value in the other locations. These values are averages taken over many samples.

\begin{tabular}{|l|c|c|c|}
\hline Location & Z & NS & EW \\
\hline Galerie Anti-Souffle & $73 \%$ & $83 \%$ & $67 \%$ \\
Galerie Gaz-Brules & $73 \%$ & $75 \%$ & $75 \%$ \\
\hline
\end{tabular}

We rescale the signal measured the Oxford system $B_{o x}$ to fit the $[\mathrm{SQUID}]^{2}$ data $B_{[S Q]}$ for the same period as follows

$$
B_{o x}^{\prime}=\alpha B_{o x}+\beta \text {. }
$$

Where $\alpha$ and $\beta$ are calculated to minimise $\sum\left(B_{o x}^{\prime}-B_{[S Q]}\right)^{2}$. This approach is explained in more detail in reference 2 . The parameter $\beta$ gives the DC offset between the two datasets; as SQUIDs measure only relative changes in the field this is entirely arbitrary. (The DC offset on the SQUID signal will change whenever the SQUIDs are tuned). The parameter $\alpha$ then gives the average ratio of the signal fluctuations over this period.

$$
\alpha_{C A P}=\frac{\left\langle B_{C A P}^{[\mathrm{SQ}]}\right\rangle}{\left\langle B_{C A P}^{\mathrm{ox}}\right\rangle} \quad \text { and } \quad \alpha_{G A S}=\frac{\left\langle B_{C A P}^{[\mathrm{SQ}]}\right\rangle}{\left\langle B_{G A S}^{\mathrm{ox}}\right\rangle} .
$$

Where $\alpha_{C A P}$ is calculated for the data set with the Oxford system in the Capsule, and $\alpha_{G A S}$ is calculated for the data set with the Oxford system in the galerie anti-Souffle. Comparing the expressions above gives

$$
A_{G A S}=\frac{\left\langle B_{C A P}\right\rangle}{\left\langle B_{G A S}\right\rangle}=\frac{\alpha_{G A S}}{\alpha_{C A P}} .
$$

Taking the $\alpha$ values in Table 1 we calculate the ratios in Table 2 . We stress that this is a preliminary analysis and the accuracy of these figures is unknown. This process is illustrated in Figure 5 which shows two samples of data recorded in the capsule and galerie anti-souffle. Table 2 gives the average attenuation determined from many samples. If these values are accurate it suggests the additional rock and steel surrounding the capsule attenuates the field to about $75 \%$ of the value in the other locations.

To determine the absolute shielding factor of LSBB (the ratio of the magnetic field outside the laboratory, to that inside) we also took measurements just outside the main entrance. The data taken here was very noisy, with many resets and flux jumps, however we are optimistic that it may be possible to find a sufficient number of stable periods to calculate this. We also intend to compare the field measured in the Capsule to that from nearby geomagnetic observatories.

This analysis assumes that the magnetic field within the capsule does not vary over the $\sim 1 \mathrm{~m}$ distance between the two magnetometers. Further investigation is necessary to determine the magnitude of this variation. We have also assumed there is no significant frequency dependence in the shielding factor over the quasi-dc regime which we are studying. This is also likely to be false, and we indeed to do a 
V. Andrieux et al.: Characterisation of magnetic field fluctuations at different locations
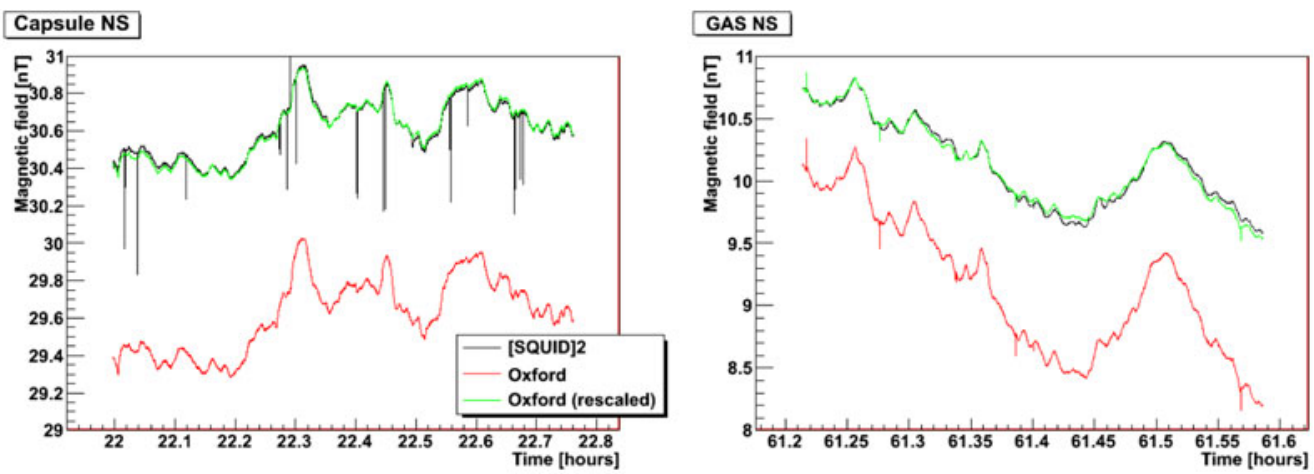

Figure 5. An illustration of the process to rescale the Oxford data to fit the [SQUID] $]^{2}$ data. The lower red line shows the Oxford data $\left(B_{o x}\right)$ recorded in the Capsule (left) and the Galerie Anti-souffle (right). The upper black line shows the $[\mathrm{SQUID}]^{2}$ measurement in the Capsule, and superimposed on this in green is $0.804 B_{o x}+6.79$ (left) and $0.620 B_{o x}+4.45$ (right). As the absolute value is arbitrary the offset between traces has been adjusted for clarity. From that we conclude the field in the capsule is attenuated by a factor of $0.620 / 0.804$ that in the GAS. Table 2 gives the value of this attenuation averaged over many such samples.
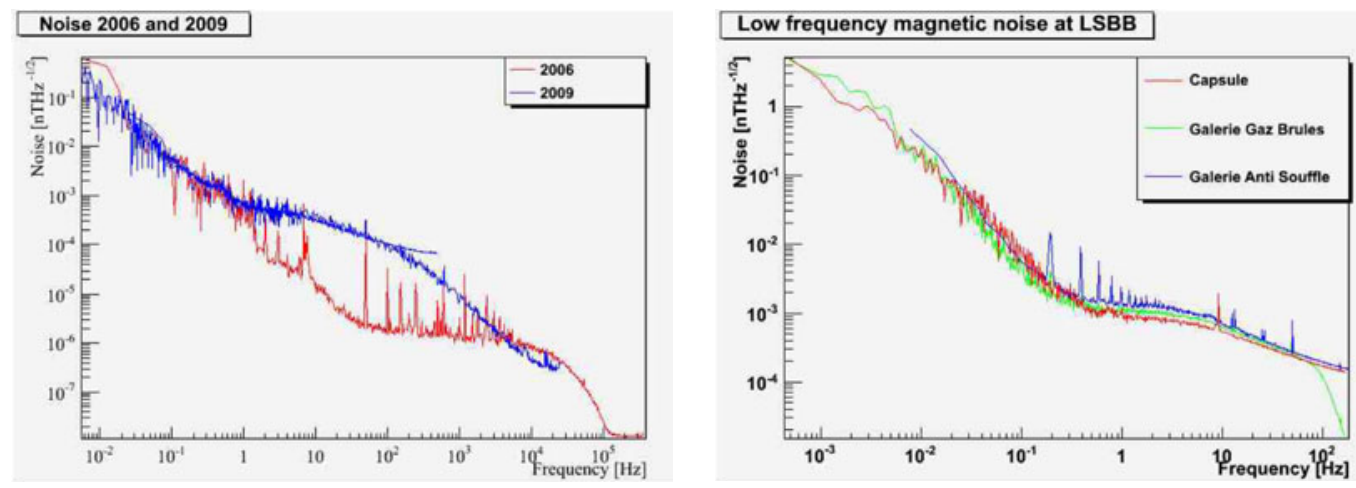

Figure 6. Noise spectra measured by the Oxford SQUID magnetometer at LSBB (the channel measuring the Zfield component, data recorded 25 September 2009). Left: comparison of the noise measured in 2006 and 2009. The higher noise above $1 \mathrm{~Hz}$ measured in 2009 is believed to be a feature of the SQUID system used, due to eddy currents in the copper former holding the pick-up loops. Right: comparison of the noise measured in different locations at LSBB. Below $0.1 \mathrm{~Hz}$ this is the spectrum of the external magnetic field, at the same level as that measured with the [SQUID] $]^{2}$ system [5].

further study of the frequency dependence once more data are available. We have neglected any pick-up of orthogonal field components, which may also be significant. ${ }^{4}$

\subsection{Noise spectra}

Figure 6 shows the noise spectral density (in $n T \cdot H z^{-1 / 2}$ ) measured using the 3 -axis setup. Below $1 \mathrm{~Hz}$ the measured noise is the magnetic field noise due to the geomagnetic field. Above $1 \mathrm{~Hz}$ the intrinsic noise of the SQUID dominates. This intrinsic SQUID noise measured in 2009 was significantly higher compared to our earlier measurements in 2006. We believe this because the pick-up loops used in 2009

\footnotetext{
${ }^{4}$ We assume the Z,NS,EW channels just measure the component they are intended to, however an alignment error or imperfect geometry of the pick-up loops would cause them to pick-up other components.
} 
i-DUST 2010

were wound on a copper former, whereas the 2006 loops were wound on a plastic former. The extra noise above $1 \mathrm{~Hz}$ is believed to be due to eddy currents in the copper former holding the pick-up loops. This did not significantly affect the measurements outlined above as these concern the low frequency region.

The right hand plot on Figure 6 shows the noise measured in different locations at LSBB. The magnitude is very similar for all locations. The Galerie Anti-Souffle plots show a series of peaks below $1 \mathrm{~Hz}$ which may be due to vibrations. At this location the helium dewar containing the magnetometer was placed directly on the gravel floor; whereas at other locations it was placed in a sandbox standing on the concrete floor.

Note that this plot cannot be compared with the attenuation factors calculated in the previous section as this analysis was done for a different frequency range (quasi-dc fluctuations). As the noise plotted for the Capsule is less than that for the Galerie Gaz-Brules below $5 \times 10^{-3} \mathrm{~Hz}$, this could support the attenuation factors given above, but there are too few data points to be sure.

\section{CONCLUSIONS}

This report outlines the measurements we have taken at LSBB with the SQUID magnetometer developed by Oxford University for the cryoEDM experiment. We have successfully shown that our upgraded system can monitor the magnetic field, and the continuous digitisation works well, and ensures that no SQUID resets are missed. We have used the data taken to develop software to correct SQUID resets.

Measurements were taken in a number of different locations and we plan to use these data to determine the relative magnetic field strengths in different locations, by comparing our signal with that measured by the $[\text { SQUID }]^{2}$ system. We have presented some preliminary results in section 3 . For a variety of reasons, given in the previous section we do not believe these figures are accurate; we present these results as a preliminary description of an analysis which we plan to do once we have collected further data and investigated systematic effects in more detail.

Noise spectra plotted from our data showed no significant difference in the magnetic field noise measured in the capsule, the galleries anti-souffle, and the galerie gaz-brulés. The dominant noise above $1 \mathrm{~Hz}$ was produced by our system.

\section{References}

[1] CA Baker et al. Phys. Rev. Lett. 97, 131801 (2006)

[2] S. Henry et al. J. Instrum. 3, P11003 (2008)

[3] http://lsbb.oca.eu/spip.php?article129

[4] G. Waysand et al. Eur. Phys. J-Appl. Phys. 47, 12705 (2009)

[5] http://1sbb.oca.eu/spip.php?article25 